\title{
Evidence on a link between the intensity of Schumann resonance and global surface temperature
}

\author{
M. Sekiguchi ${ }^{1}$, M. Hayakawa ${ }^{1}$, A. P. Nickolaenko ${ }^{2}$, and Y. Hobara ${ }^{1,3}$ \\ ${ }^{1}$ The University of Electro-Communications, Department of Electronic Engineering, Chofu, Tokyo, Japan \\ ${ }^{2}$ Usikov's Institute for Radiophysics and Electronics, National Academy of Science of the Ukraine, Kharkov, Ukraine \\ ${ }^{3}$ Swedish Institute of Space Physics, Kiruna, Sweden
}

Received: 19 August 2005 - Revised: 12 June 2006 - Accepted: 14 June 2006 - Published: 9 August 2006

\begin{abstract}
A correlation is investigated between the intensity of the global electromagnetic oscillations (Schumann resonance) with the planetary surface temperature. The electromagnetic signal was monitored at Moshiri (Japan), and temperature data were taken from surface meteorological observations. The series covers the period from November 1998 to May 2002. The Schumann resonance intensity is found to vary coherently with the global ground temperature in the latitude interval from $45^{\circ} \mathrm{S}$ to $45^{\circ} \mathrm{N}$ : the relevant crosscorrelation coefficient reaches the value of 0.9 . It slightly increases when the high-latitude temperature is incorporated. Correspondence among the data decreases when we reduce the latitude interval, which indicates the important role of the middle-latitude lightning in the Schumann resonance oscillations. We apply the principal component (or singular spectral) analysis to the electromagnetic and temperature records to extract annual, semiannual, and interannual variations. The principal component analysis (PCA) clarifies the links between electromagnetic records and meteorological data.
\end{abstract}

Keywords. Meteorology and atmospheric dynamics (Atmospheric electricity; Climatology) - Radio science (Remote sensing)

\section{Introduction}

A correspondence was demonstrated by Williams (1992) between the long-term Schumann resonance amplitude and the temperature anomaly in the tropical belt. The global electromagnetic or Schumann resonance is a phenomenon that takes place in the spherical Earth-ionosphere cavity (Nickolaenko and Hayakawa, 2002). Oscillations are maintained by the global lightning activity, which radiates the extremely low frequencies (ELF). The intensity of the resonance reflects

Correspondence to: M. Hayakawa

(hayakawa@whistler.ee.uec.ac.jp) the instantaneous level of thunderstorm activity, and it must vary with the global temperature. Williams (1992) used the Schumann resonance records collected by Charles Polk at the Rhode Island field site; the duration covered six years (Polk, 1969, 1982). We show in Fig. 1 the plot adopted from Williams (1992), where the electromagnetic data are compared with the monthly mean fluctuation in the surface (drybulb) temperature for the entire tropics. Time is shown on the abscissa, the global temperature anomaly in the tropical region is shown along the right ordinate in centigrade (the heavy line), and the amplitude is plotted on the left ordinate measured in the horizontal magnetic field at the fundamental mode of Schumann resonance (the line with open squares). As one may see, Fig. 1 shows that the Schumann resonance amplitude follows the temperature anomaly quite closely for the long-period variation.

An interest in the Schumann resonance reappeared in the 90s. Many observatories were set at work (Sentman, 1995; Satori and Zieger, 1996; Füllekrug and Fraser-Smith, 1997; Nickolaenko, 1997; Nickolaenko et al., 1998, 1999; Heckman et al., 1998; Hobara et al., 2000, 2001, 2003; Hayakawa et al., 2004), and noticeable progress was made in the Schumann resonance modeling (Kirillov et al., 1997; Hayakawa and Otsuyama, 2002; Mushtak and Williams, 2002; Otsuyama et al., 2003; Simpson and Taflove, 2004; Yang and Pasko, 2005). The present work is a further development of the idea by Williams (1992), Price (1993), Füllekrug and Fraser-Smith (1997), and Williams (1994). We investigate the relationship between the Schumann resonance intensity and the global soil temperature, the latter was measured in symmetric latitudinal intervals. The driving idea is rather straightforward. Electromagnetic radiation from the global lightning strokes is the source of electromagnetic energy detected in the Schumann resonance band (from a few $\mathrm{Hz}$ to some tens of $\mathrm{Hz}$ ). The planetary thunderstorm activity is concentrated in the tropics. The lightning flash rate is collected from space, using the optical transient detector

Published by Copernicus GmbH on behalf of the European Geosciences Union. 


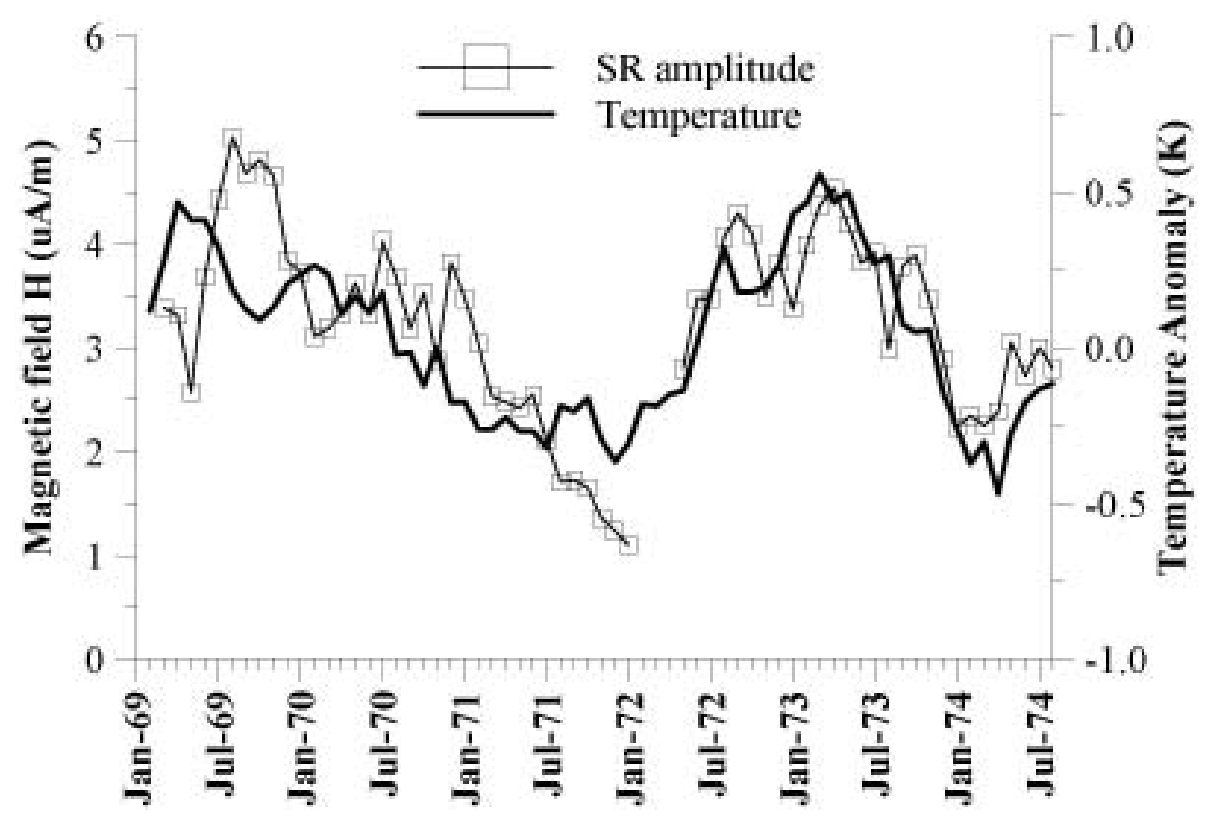

Fig. 1. Correlation between the global temperature and the intensity of Schumann resonance oscillations (adopted from Williams, 1992).

(OTD) (Orville and Henderson, 1986; Christian et al., 2003; Hayakawa et al., 2005). The lightning flash is high in the tropics, remains relatively high in the mid-latitudes, and becomes rare in the polar and sub-polar regions. Therefore, one may expect that the Schumann resonance intensity is connected with the surface temperature within a certain latitudinal interval. We are going to test this idea and establish the width of such an interval.

By following the Sun, global thunderstorms cyclically drift along the meridian to the Northern Hemisphere during boreal summer, and drift southward from the equator in winter. Since the continents occupy a greater area in the Northern Hemisphere, an annual variation develops in the thunderstorm activity: the global flash rate is smaller during the winter months (Christian et al., 2003; Hayakawa et al., 2005). Therefore, annual variations must also be present in the Schumann resonance intensity. The goal of our investigation is a formal comparison of trends present in the Schumann resonance intensity and the ground temperature itself.

We correlate the long-term seasonal variations of cumulative intensity of three Schumann resonance modes with the changes in the median global land temperature relevant to different symmetric latitudinal belts. We look for the interval where the close correlation appears between the climatological and electromagnetic data. Afterwards, to remove the random oscillations always present in the data sets, we turn to the principal components (Troyan and Hayakawa, 2003), namely, to the annual, semiannual and interannual trends, which are extracted from the raw temperature and Schumann resonance data. Finally, the current results are discussed and explained, and the areas of future work are outlined.

\section{Acquisition of Schumann resonance data and signal processing}

The natural ELF signal is monitored at the Moshiri observatory (Japan, $44^{\circ} 22^{\prime} \mathrm{N}$ and $142^{\circ} 15^{\prime} \mathrm{E}$ ) since 1996. Two orthogonal horizontal magnetic field components $\left(\mathrm{H}_{N S}\right.$ and $\left.\mathrm{H}_{E W}\right)$ and one vertical electric field component $\left(\mathrm{E}_{Z}\right)$ are recorded simultaneously; the details are found in Hobara et al. (2001). In this paper, we discuss the continuous time series of the horizontal $\mathrm{H}_{N S}$ field component, covering the period from November 1998 to May 2002. The longest continuous data set is available in this particular component (with a few data gaps). The other components, unfortunately, have greater gaps caused by the local interferences or by the temporal system malfunction. In addition, the $\mathrm{E}_{Z}$ records suffer from the bad weather conditions.

The notation of $\mathrm{H}_{N S}$ corresponds to the magnetic coil antenna aligned to the local meridian; such a sensor is sensitive to the radio waves arriving from the east (thunderstorms in America) or from the west (African activity). The ELF measurement system is periodically calibrated.

The power spectra of the Schumann resonance are computed during the processing of a record with the FFT algorithm. Spectra are averaged over segments 10 min long. These data are additionally averaged over each hour of the day, which allows us to reduce the impact of data gaps on the output results. Finally, the monthly mean is computed for every hour. Thus, we obtain the monthly averaged spectra as the function of universal time. These sets were ultimately 


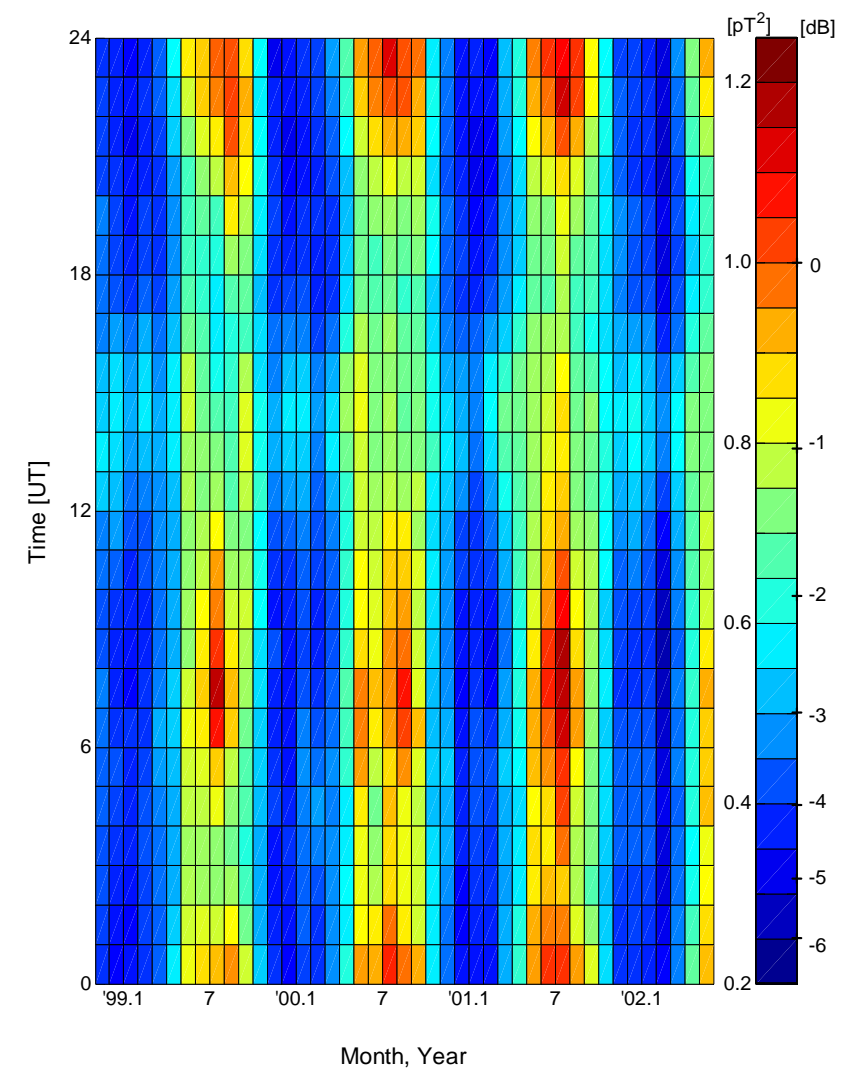

Fig. 2. Monthly averaged diurnal variation of the cumulative energy of Schumann resonance. The color code is used to indicate the absolute intensity (in $\mathrm{pT}^{2}$ ) (and also in $\mathrm{dB}$ ).

averaged to produce the median Schumann resonance spectra relevant to each month of a year.

Schumann resonance is observed as the peaks (modes) in the power spectrum of the given field component. Each mode corresponds to a particular spatial distribution of the field. Thunderstorms move around the globe during the day, the distance from an observatory to the lightning sources varies, and the amplitude of individual Schumann resonance modes also varies. Alterations caused by the source position are superimposed on the changes connected with the contemporary intensity of the global thunderstorm activity. So, when obtaining the estimates of thunderstorm intensity, we have to reduce the impact of distance variations. The cumulative intensity of three Schumann resonance modes is used for this purpose, being less sensitive to the source-observer distance (see Polk, 1969, Sentman and Fraser, 1991; Nickolaenko, 1997, Nickolaenko et al., 1999, Nickolaenko and Hayakawa, 2002):

$I=A_{1}^{2}+A_{2}^{2}+A_{3}^{2}$.

Here, $A_{i}$ is the magnetic field amplitude of the $i$-th mode $(i=1,2,3)$. We use the $\pm 0.5-\mathrm{Hz}$ bandwidth for each mode. This approach was exploited in the experimental studies of
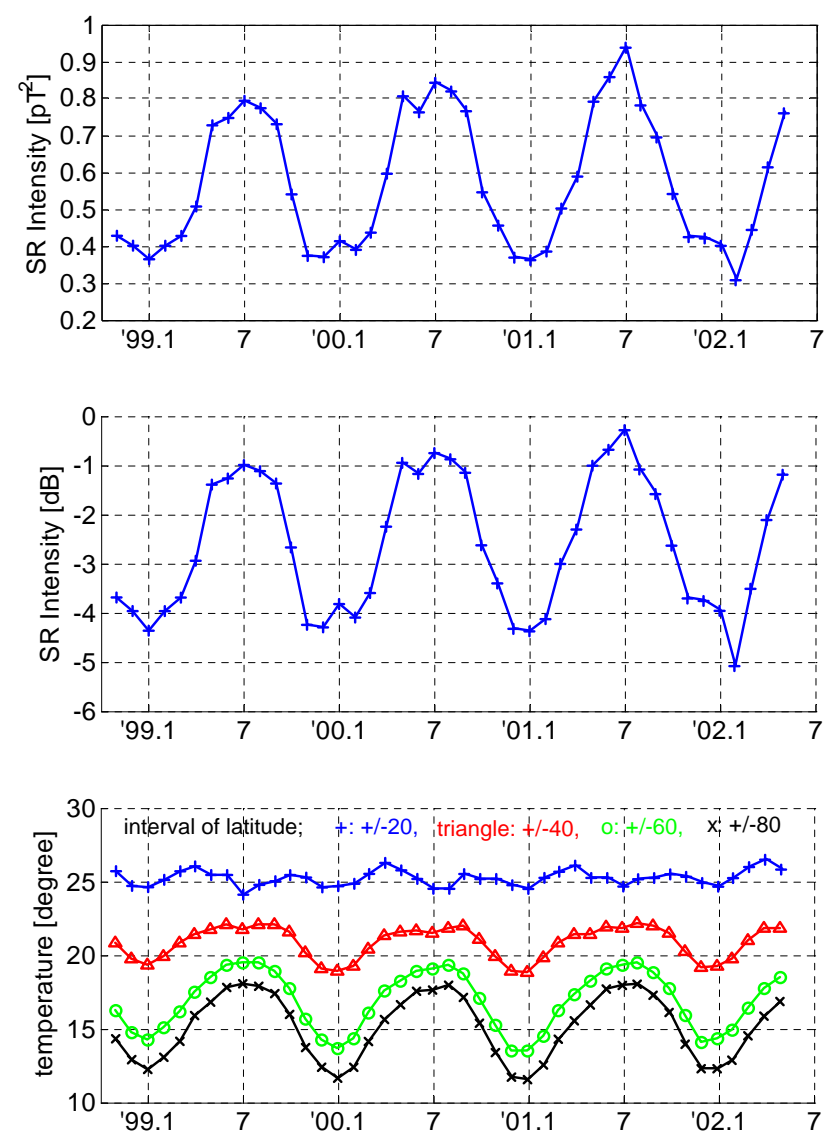

Fig. 3. Seasonal variations of electromagnetic and temperature data. Top and middle panels show the Schumann resonance intensity in $\mathrm{pT}^{2}$ and in $\mathrm{dB}$, correspondingly. Bottom panel presents the global mean ground temperature in symmetric latitudinal belts $\pm 20^{\circ}, \pm 40^{\circ}, \pm 60^{\circ}, \pm 80^{\circ}$.

Schumann resonance when obtaining estimates for the instant level of global thunderstorm activity (Clayton and Polk, 1997; Heckman et al., 1998; Nickolaenko et al., 1998, 1999).

Figure 2 illustrates the dynamics of the monthly averaged daily variation of the Schumann resonance intensity (1) during the whole period of observations. The abscissa indicates the month and year, while the ordinate indicates the universal time (UT). The measuring equipment is fully calibrated (Hobara et al., 2000), and the presentation in Fig. 2 is given in the absolute intensity $\left(\mathrm{pT}^{2}\right)$ and in the $\mathrm{dB}$ relative to $1 \mathrm{pT}^{2}$ : $I[\mathrm{~dB}]=10 \lg \left(I / I_{R}\right)$, where $I_{R}=1 \mathrm{pT}^{2}$. The absolute intensity observed is in accord with the published data (see Sentman (1995)). The strong American thunderstorm activity is observed in the plots of Fig. 2 around midnight (23:00-00:00 hr) UT. A smaller African activity is visible at 14:00-15:00 UT, being somewhat reduced by the angular pattern of the magnetic antenna. An impact of the nearby powerful storms in Southeast Asia is present at 7:00-10:00 UT, even in the $H_{N S}$ field component. 


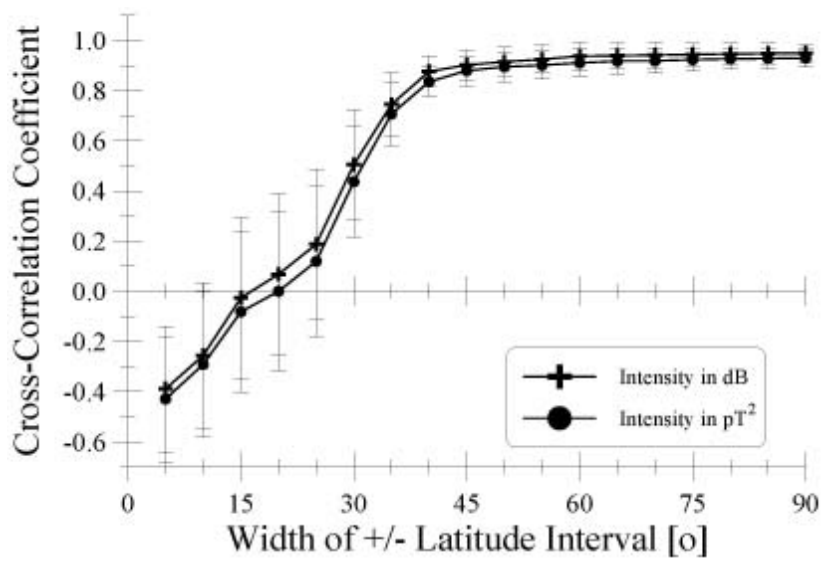

Fig. 4. Cross-correlation coefficient as a function of the latitude interval, for the intensity measured in absolute values (line connected by crosses) and for the intensity measured in $\mathrm{dB}$ (line connected by circles). The error bar is also indicated (as the level of confidence of 0.95$)$.

The initial data sets are presented in Fig. 3. The upper panel in Fig. 3 depicts the absolute power (in $\mathrm{pT}^{2}$ ) of the three Schumann resonance modes (1) as a function of time recorded at Moshiri station from November 1998 to May 2002. The second frame repeats the same plot translated to $\mathrm{dB}$ relative to the $1 \mathrm{pT}^{2}$ scale. The bottom panel presents seasonal variations of the global land surface temperature in different latitudinal intervals. Here the time (in months) is plotted on the abscissa. Vertical dotted lines mark the halfyear intervals. As one may see from Fig. 3, the annual variation of the Schumann resonance intensity is close to $5 \mathrm{~dB}$ (amplitude variation by a factor of two).

\section{Surface temperature data}

The bottom panel of Fig. 3 depicts seasonal variations of the surface temperature computed within intervals ranging from $20^{\circ} \mathrm{S}$ to $20^{\circ} \mathrm{N} ; 40^{\circ} \mathrm{S}-40^{\circ} \mathrm{N} ; 60^{\circ} \mathrm{S}-60^{\circ} \mathrm{N}$; and $80^{\circ} \mathrm{S}-80^{\circ} \mathrm{N}$. The data were selected from the National Climate Data Center (USA), which provides monthly mean temperatures for the land area based on the surface meteorological observations (see Petersen and Vose, 1997). Initial data correspond to the grid $15^{\circ} \times 5^{\circ}$ (longitude times latitude, respectively) covering the entire Earth. The grid mean temperature was calculated for each interval: mean temperature at given latitudes was obtained by averaging the grid data in accordance with the ground area found in the interval.

To reduce the impact of seasonal north-south drift, we averaged the temperature data in the symmetric latitude intervals centered at the equator. Thus, we consistently include the surface temperature of the most important tropical belt, corresponding to the narrow central peak in the latitudinal distribution of lightning flashes observed from space (Chris-
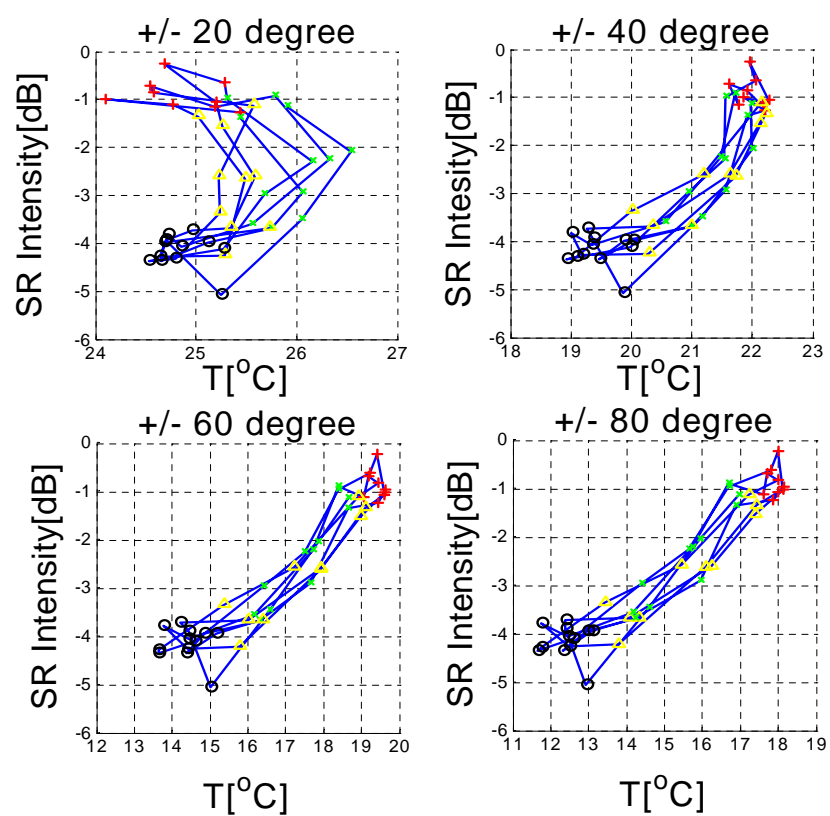

Fig. 5. Lissajous figures of the Schumann resonance intensity versus global temperature. Green markers denote spring, red is summer, yellow is autumn, and black shows winter.

tian et al., 2003; Hayakawa et al., 2005). As might be concluded from Fig. 3, this zone is associated with the strong semiannual variation. The north-south seasonal drift and the asymmetry in the global land distribution appear as the annual variation in the surface temperature (see the lower plots in Fig. 3 for wide latitudinal gaps). The semiannual variation is always present; however, it seems to diminish in the annual variation pertinent to the middle latitudes. The median temperature decreases when the width of the latitudinal interval increases, reflecting the general decline of the temperature with latitude.

\section{Correlation between Schumann resonance intensity and global temperature}

We calculated the cross-correlation coefficients for variations of the Schumann resonance intensity and the global ground temperature averaged in different latitudinal belts; see Fig. 4 (the widths of $\pm 5^{\circ}, \pm 10^{\circ}, \pm 15^{\circ}$, etc., were used). The width of latitude interval is shown on the abscissa of Fig. 4 in degrees, and the cross-correlation coefficient is indicated on the ordinate. It seems that a systematically stronger correlation is apparent between the temperature and the Schumann resonance intensity measured in $\mathrm{dB}$ (crosses) rather than absolute intensity (circles). This property implies that a link between the global temperature and electromagnetic intensity $I$ is of the following form: $I=<|\mathrm{H}|^{2}>=I_{0} \cdot \exp \left[\zeta\left(T-T_{0}\right)\right]$, where $T$ is current temperature and $T_{0}$ is its median value for the given 
latitudinal belt. It is clear that the field level in $\mathrm{dB}$ is a measure of the exponent $\zeta$.

One may see from Fig. 4 that the cross-correlation coefficient becomes saturated when the latitude interval exceeds $\pm 45^{\circ}$. Such a behavior might be expected, since the lightning activity is concentrated in the tropics and sub-tropics. On the other hand, a contribution from the mid-latitude temperature into the Schumann resonance intensity (and hence the role of the mid-latitude lightning activity) is significant. The conclusion is supported by the upper frames of Fig. 3, showing a strong annual variation in the Schumann resonance intensity, while the semiannual component prevails in the tropics and sub-tropics. This is the reason why two processes have insignificant cross-correlation until the latitude interval becomes sufficiently wide.

The connection between two periodic variables, including their mutual phase shift is distinctly demonstrated by the Lissajous plots shown in Fig. 5. Here, the seasonal variations of the global soil temperature are plotted on the abscissa. The ordinate shows the Schumann resonance intensity in $\mathrm{dB}$. We use various colors to show different seasons: green marks the spring, the red marks the summer, yellow is autumn, and black is winter. It is easy to see from Fig. 5 that the Schumann resonance intensity and global ground temperature tend to vary coherently. However, their links are complicated by the presence of higher harmonics and the phase shifts.

\section{Periodic variations of Schumann resonance intensity and of the global temperature}

The pattern of seasonal variation of the surface temperature depends on the width of the latitude interval. It consists of two major components: one is the semiannual variation coupled to the tropics, and the other is the annual component, corresponding to a wider latitude band. There are both annual and semiannual components present in the longterm variations of Schumann resonance parameters. A strong semi-annual variation was found in the frequency of the first resonance mode observed in the vertical electric field component $E_{Z}$, which clearly reflects the seasonal variations of the area covered by the global lightning activity (Nickolaenko et al., 1998). A semiannual variation was also found in the cumulative field intensity recorded in the late 60s in Japan (Nickolaenko et al., 1999). It is interesting to use a similar process for the modern Schumann resonance records and compare the results with the previous data and with concurrent variations in the global temperature.

We apply the Principal Component Analysis (PCA), which is also regarded in the literature as singular spectral analysis (SSA), to extract periodic components from the Schumann resonance intensity $(\mathrm{dB})$ and from the net global temperature in different latitudinal belts. The software is called the "Caterpillar" algorithm (Troyan and Hayakawa, 2003). We extract the unknown regular variations which are hidden in the record, and the PCA algorithm is an appropriate tool for this purpose (Danilov and Zhiglyavsky, 1997; Troyan and Hayakawa, 2003). From the physical point of view, the PCA algorithm is similar to filtering, although its main distinction is that the basic functions are found automatically right from the original data.

The algorithm works in the following way. Consider a finite time series of initial data $x_{k}=x\left(t_{k}\right)$ with $1 \leq k \leq N$. An integer number $L<N$ is chosen, called the "caterpillar" length, and the linear set $x_{k}$ is transformed into a 2-D matrix in the following way. The first $L$ points of the $x_{k}$ series (we use $L=12$, corresponding to the annual period) occupy the first line of the matrix. The elements from $x_{2}$ to $x_{L+1}$ are placed in the second line, etc. The process continues until we reach the end of the data. The last line of the matrix contains the elements with indices starting from $N-L+1$, so that the last $L$ samples are, $x_{N-L+1}, x_{N-L+2}, \ldots, x_{N}$.

The eigen-values and eigen-vectors are found for the 2-D matrix in the second step of processing. These parameters depend on the signal structure and they allow us simultaneously to construct the "internal basis" relevant to the data: the so-called principal components. This stage of the procedure is equivalent to composing the bank of linear filters. Each filter corresponds to a particular principal component. We emphasize that filters are found from the data itself rather than postulated beforehand.

In the third step, we visualize, survey, and select the desired principal components (PC) revealed in the previous step. The periodic principal components split into pairs: one of them resembles the "sine" wave, while the other is the "cosine" wave. We usually take PC \#1 and PC\#2 to obtain the complete annual variation of the lowest frequency.

The final step implies the signal reconstruction by selecting and combining the desired principal components. This step is similar to extracting a set of harmonics in the conventional Fourier transform. We must add that the PCA procedure turns into an ordinary Fourier transform when the initial succession $x_{k}$ is a sinusoidal signal of infinite duration. In this case, the matrix constructs authentic sine and cosine basic functions, and the result coincides with the well-known procedures (Danilov and Zhiglyavsky, 1997).

To obtain both annual and semiannual components of the variations of global temperature and Schumann resonance intensity, we apply the PCA procedure with the length $L=12$ (one year) and concentrate our attention on PC\#1 and PC\#2 (annual variation) and PC\#3 and PC\#4 (semiannual term). The annual component is present in both temperature and Schumann resonance intensity. A substantial semiannual variation is found only in the global temperature of the tropical belt.

The PCA processing also shows that initial data contain only these two major components, plus insignificant random fluctuations, and we summarize the results in Table 1. The first column of this table denotes the variable. Column 
Table 1. Annual and semiannual components extracted from the data series by the PCA processing.

\begin{tabular}{|c|c|c|c|c|}
\hline \multirow{2}{*}{$\begin{array}{l}\text { Variable } \\
\text { SR intensity }\end{array}$} & \multicolumn{2}{|c|}{ Annual components, $\%$} & \multicolumn{2}{|c|}{ Semiannual components, $\%$} \\
\hline & $\mathrm{PC} \# 1=49.5$ & $\mathrm{PC} \# 2=45.6$ & $\mathrm{PC} \# 3=1.2$ & $\mathrm{PC} \# 4=1.1$ \\
\hline Temperature in $\pm 20^{\circ}$ interval & $\mathrm{PC} \# 3=9.5$ & $\mathrm{PC} \# 4=8.2$ & $\mathrm{PC} \# 1=34.6$ & $\mathrm{PC} \# 2=33.7$ \\
\hline Temperature in $\pm 40^{\circ}$ interval & $\mathrm{PC} \# 1=43.5$ & $\mathrm{PC} \# 2=40.6$ & $\mathrm{PC} \# 3=7.0$ & $\mathrm{PC} \# 4=6.7$ \\
\hline Temperature in $\pm 60^{\circ}$ interval & $\mathrm{PC} \# 1=49.0$ & $\mathrm{PC} \# 2=46.1$ & $\mathrm{PC} \# 3=2.1$ & $\mathrm{PC} \# 4=2.0$ \\
\hline
\end{tabular}

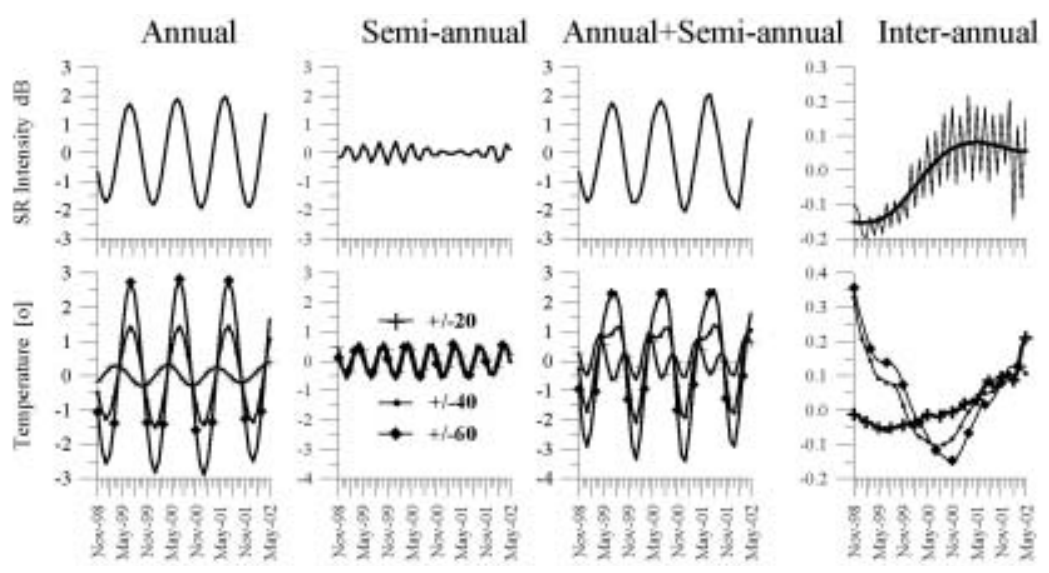

Fig. 6. Principal components: annual, semiannual, annual + semiannual, and interannual. The upper plots refer to the variations of the Schumann resonance intensity, and the lower plots demonstrate the variations of the global soil temperature in different latitude intervals.

numbers 2 and 3 refer to an annual variation, and columns 4 and 5 correspond to a semiannual component. The numbers indicate the contributions of particular principal components into the energy of initial variation. Table 1 indicates that the semiannual term is very small in the Schumann resonance intensity: its contribution is below $3 \%$, while the annual variation is responsible for $95 \%$. The semiannual contribution is considerably smaller than in our previous study (about 20\%), based on the old resonance data collected in Japan (Nickolaenko et al., 1999). In contrast, Table 1 shows that the annual pattern in the land temperature is of minor importance within the $\pm 20^{\circ}$ latitude gap: its contribution is $17 \%$ against $68 \%$ from the semiannual trend.

We depict temporal variations of the major principal components in Fig. 6. Time in months is shown on the abscissa. The ordinate depicts the resonance intensity in $\mathrm{dB}$ (upper plots) and temperature in centigrade (lower plots). By comparing Figs. 6 and 3, one may observe how the PCA processing "rectified" the variations. Figure 6 presents the annual trends (the left frames), semiannual terms (the second column of plots), the composition of annual and semiannual variations (the third plot), and interannual variation (the right frames). As is shown in the lower plots of Fig. 6, the amplitude of the annual temperature variation increases when a wider latitude interval is analyzed. This is explained by an increase in the north-south asymmetry of the land area in wider latitude intervals. Variations in the $\pm 40^{\circ}$ and $\pm 60^{\circ}$ belts occur in phase, while the temperature pattern of $\pm 20^{\circ}$ latitudinal belt leads in phase by approximately 3 months. Contribution from the polar latitudes into annual variations of global temperature is insignificant (not shown), as was expected. However, the middle latitudes (from 40 to $60 \mathrm{deg}$ ) are responsible for a substantial fraction in annual variation of the global land temperature. Amplitude and phase of the semiannual component remain unchanged when we extend the interval of latitudes, as is seen in the second lower plot of Fig. 6. This means that only the tropical region contributes to this semiannual signal.

Plots of Fig. 6 enable us to observe the important features:

1. Variations of electromagnetic intensity and land temperature on the annual scale are similar in the wide latitude belts.

2. The beating mode is observed in the semiannual Schumann resonance data, which agrees with the published results.

3. Semiannual temperature variations coincide for all the belts, which means that this term originates from the tropical region.

4. General variations of the Schumann resonance intensity occur in the way similar to the temperature observed in 


\section{Initial Data}
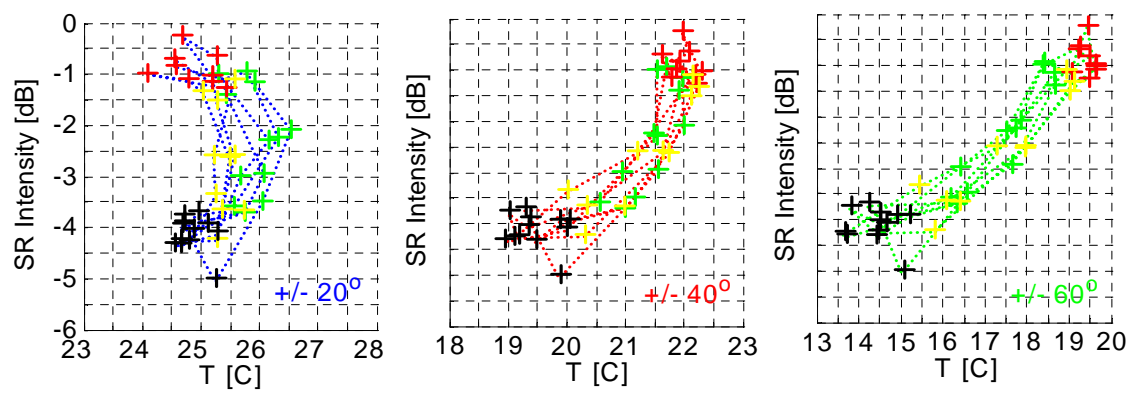

annual vs Annual Component
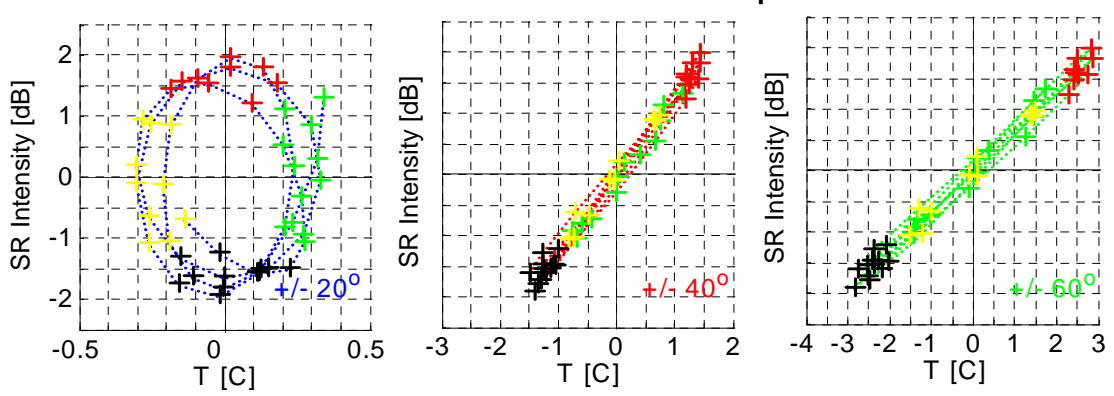

Annual vs Annual+Semi-Annual Component
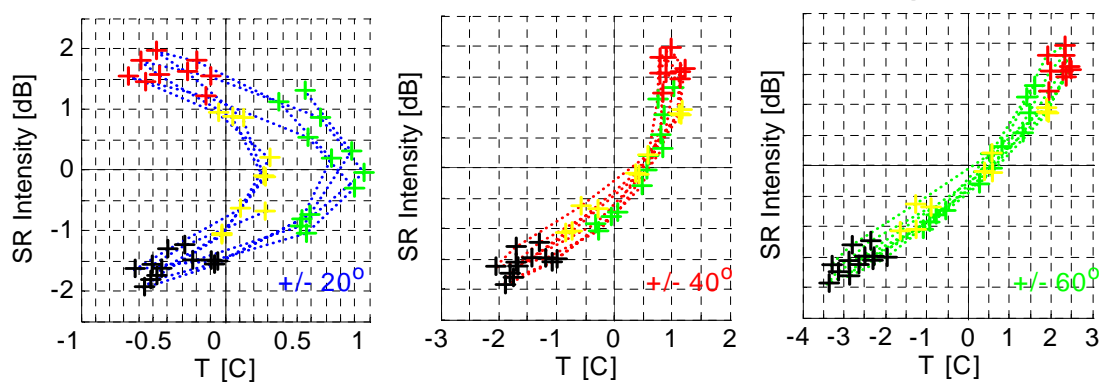

Fig. 7. Lissajous figures of global land temperature versus Schumann resonance intensity. The color of plot indicates season (green: spring, red: summer, yellow: autumn, and winter: black).

the middle latitudes. In contrast, the interannual variations (see the right plots in Fig. 6) exhibit a similarity between the Schumann resonance data and the temperature trends of the topical region. Unfortunately, the 3.5-year duration of the data is somewhat short to extract the interannual alterations with confidence and to make decisive conclusions, however, the similarity must be noted.

We may expect that Lissajous figures will vary when we compare electromagnetic energy with the land temperature of different latitude belts. We show in Fig. 7 the Lissajous patterns of the initial data (the upper line of plots), the annual trends (the middle plots), and composition of annual and semiannual components (the lower plots). The Schumann resonance intensity is shown on the ordinate of each frame in $\mathrm{dB}$. Variations of temperature are plotted on the abscissa. The left column of panels relates the variations of the Schumann resonance intensity with temperature in the trop- ical $\pm 20^{\circ}$ region. The central plots correspond to a $\pm 40^{\circ}$ latitude interval, and the right column depicts the data for a $\pm 60^{\circ}$ belt.

The left frame in the middle row shows that the annual component of temperature in $\pm 20^{\circ}$ latitudes is in advance by about three months with respect to the annual variations of Schumann resonance intensity. The plot is practically a circle. Annual temperature variations in wider belts occur in phase with the electromagnetic data, and we observe the straight line in the central and right frames of the middle row. The lower plots depict the sums of the annual and semiannual components extracted from the records. The left plot reminds us of a typical Lissajous figure for two sinusoidal signals, with the frequency ratio $2: 1$. The central and right plots are just the same in-phase variations of the middle row, but slightly "spoiled" by the "second harmonic". 


\section{Discussion and conclusions}

We processed the modern long-term records of the Schumann resonance, which have the duration comparable with the former data collected by Prof. K. Sao's group in the Tottori observatory (Japan) in the 70s (Nickolaenko et al., 1999). The same software was applied toward the new data set. The annual component of the Schumann resonance intensity in the modern records is in good correspondence to that found in the old records. The complete annual excursion of the Schumann resonance intensity is about $5 \mathrm{~dB}$ for the $H_{N S}$ field observed in Japan. Characteristic variation extracted by the PCA algorithm is somewhat smaller, about $\pm 2 \mathrm{~dB}$. The semiannual component is smaller by an order of magnitude, and this is the main distinction of the new record from the previous data. We cannot indicate the reason of deviation; however, the data of the 70s contained a strong temporal modulation of semiannual pattern, the "beating mode". It might be that recent records coincide with the period of small amplitude in the semiannual term, owing to some unknown natural factor.

Variations of the Schumann resonance intensity occur in the way similar to the temperature within the middle-latitude interval. In contrast, the interannual variation of Schumann resonance resembles that of temperature in the topics (see the right plots in Fig. 6). Unfortunately, the 3.5-year duration does not allow us to make the decisive conclusions.

A comparison of electromagnetic and temperature data indicated that there is a link between the annual variation of the Schumann resonance intensity and the global temperature. The cross-correlation coefficient reaches $0.05,0.85$, 0.92 , and 0.95 when we extend the latitude intervals from $\pm 20^{\circ}$, to $\pm 40^{\circ}, \pm 60^{\circ}$, and $\pm 80^{\circ}$, correspondingly.

The data presented in this paper allow us to formulate the following conclusions.

1. Schumann resonance intensity of recent records, 43 months long in duration, made in Japan, is characterized by a strong annual variation (about $\pm 2 \mathrm{~dB}$ ), while the semi-annual trend is smaller by an order of magnitude.

2. Variations in the global land temperature are characterized by two seasonal patterns associated with different latitude intervals. The semiannual variation dominates in the tropics, and the annual trend prevails in the middle and high latitudes.

3. Intensity variations of Schumann resonance oscillations corresponds to alterations of the global land temperature in the mid-latitude interval of about $\pm 45^{\circ}$.

Finally, we mention the areas of future works in the Schumann resonance band. Simultaneous observations at a few sites (including those positioned in the Southern Hemisphere) are desirable for a reduction of source proximity ef- fects and separating contributions from thunderstorms in different latitude zones. Measurements in the Southern Hemisphere are useful, since thunderstorms drift away from the observer in the Southern Hemisphere during boreal summer, and the source distance varies in anti-phase there. The combination of records performed in both hemispheres will compensate for the meridional drift of global thunderstorms, while variations are enhanced associated with the level of global thunderstorm activity itself. Simultaneous observations at a series of stations allow for deducing the global distribution of thunderstorm activity (Shvets, 2001; Ando et al., 2005), which can also help to extract alterations in the thunderstorm activity and thus to improve the quality of initial data.

Acknowledgements. The authors would like to thank M. Sera and Y. Ikegami of the Moshiri observatory (Nagoya University) for their contribution to the ELF observation there and E. Williams of M. I. T. for his useful comments. Also thanks are due to the unknown referees for their useful comments which helped us to improve the paper.

Topical Editor U.-P. Hoppe thanks A. V. Shvets and two other referees for their help in evaluating this paper.

\section{References}

Ando, Y., Hayakawa, M., Shvets, A. V., and Nickolaenko, A. P.: Finite difference analyses of Schumann resonance and reconstruction of lightning distribution, Radio Sci., 40, no.2, doi:10.1029/2004RS003153, 2005.

Christian, H. J., Blackslee, R. J., Boccippio, D. J., Boek, W. I., Buechler, D. E., Driscoll, K. T., Goodman, S. J., Hall, J. H., Koshak, W. J., Mach, D. M., and Stewart, M. F.: Global frequency and distribution of lightning as observed from space by the Optical Transient Detector, J. Geophys. Res., 108 (D1), 4005, doi:10.1029/2002JD002347, 2003.

Clayton, M. and Polk, C. : Diurnal variation and absolute intensity of world-wide lightning activity, September 1970 to May 1971, Electrical Processes in Atmospheres, edited by: Dolezalek, H. and Reiter, R., Steinkopf, Darmstadt Germany, 111-178, 1997.

Danilov, D. L. and Zhiglyavsky, A. A. (Eds.): Principal Component of the Time Series, the Caterpillar Method (in Russian), St. Petersburg State University, St. Petersburg, Russia, 307, 1997.

Füllekrug, M. and Fraser-Smith, A. C. : Global lightning and climatic variability inferned from ELF field variations, Geophys. Res. Lett., 24, 2411-2414, 1997.

Hayakawa, M.: Satellite observation of low-latitude VLF radio noise and their association with thunderstorms, J. Geomagn. Geoelectr., 47, 573-595, 1989.

Hayakawa, M. and Otsuyama, T.: FDTD analysis of ELF wave propagation in inhomogencous subionospheric waveguide models, Appl. Computational Electromagnetics Soc. J., 17, No.3, 239-244, 2002.

Hayakawa, M., Nakamura, T., Hobara, Y., and Williams, E. : Observation of sprites over the Sea of Japan and the conditions of lightning-induced sprites in winter, J. Geophys. Res., 109, A01312, doi:10.1029/2003JA009905, 2004. 
Hayakawa, M., Sekiguchi, M., and Nickolaenko, A. P.: Diurnal variation of electric activity of global thunderstorms deduced from OTD data, J. Atmos. Electr., 25, 55-68, 2005.

Heckman, S. J., Williams, E., and Boldi, B.: Total global lightning inferred from Schumann resonance measurements, J. Geophys. Res., 103, D24, 31 775-31 779, 1998.

Hobara, Y., Iwasaki, N., Hayashida, T., Tsuchiya, N., Williams, E. R., Sera, M., Ikegami, Y., and Hayakawa, M.: New ELF observation site in Moshiri, Hokkaido, Japan and the results of preliminary data analysis, J. Atmos. Electr., 20, 99-109, 2000.

Hobara, Y., Iwasaki, N., Hayashida, T., Hayakawa, M., Ohta, K., and Fukunishi, H.: Interrelation between ELF transients and ionospheric disturbances in association with sprites and elves, Geophys. Res. Lett., 28, 935-938, 2001.

Hobara, Y., Hayakawa, M., Ohta, K., and Fukunishi, H.: Lightning discharges in association with mesospheric optical phenomena in Japan and their effect on the lower ionosphere, Adv. Polar Upper Atmos. Res., 17, 30-47, 2003.

Kirillov, V. V., Kopeykin, V. N., and Mushtak, V. C.: Electromagnetic waves of ELF band in the Earth-ionosphere waveguide (in Russian), Geomag. Aeron., 37, 114-120, 1997.

Mushtak, V. C. and Williams, E. R.: ELF propagation parameters for uniform models of the Earth-ionosphere waveguide, J. Atmos. Solar-Terr. Phys., 64, 1989-2001, 2002.

Nickolaenko, A. P.: Modern aspects of the Schumann resonance studies, J. Atmos. Solar-Terr. Phys., 59(7), 805-816, 1997.

Nickolaenko, A. P., Sàtori, G., Ziegler, V., Rabinowicz, L. M., and Kudintseva, I. G.: Parameters of global thunderstorm activity deduced from the long-term Schumann resonance records, J. Atmos. Solar-Terr. Phys., 60(3), 387-399, 1998.

Nickolaenko, A. P., Hayakawa, M., and Hobara, Y.: Long-term periodic variations in the global lightning activity deduced from the Schumann resonance monitoring, J. Geophys. Res., 104(D22), 27 585-27 591, 1999.

Nickolaenko, A. P. and Hayakawa, M.: Resonances in the EarthIonosphere Cavity, Kluwer Academic Publishers, DordrechtBoston-London, 380, 2002.

Orville, R. E. and Henderson, R. W.: Global distribution of midnight lightning: September 1977 to August 1978, Monthly Weather Rev., 114, 2640-2653, 1986.

Otsuyama, T., Sakuma, D., and Hayakawa, M.: FTDT analysis of ELF wave propagation and Schumann resonanace for a subionopheric waveguide model, Radio Sci., 38, No.6, 1103, doi:10.1029/2002RS002752, 2003.
Petersen, T. C. and Vose, R. S.: An overview of the global historical chimatology network temperature data base, Bull. Amer. Meteor. Soc., 78, 2837-2549, 1997.

Polk, C.: Relation of ELF noise and Schumann resonances to thunderstorm activity, in: Planetary Electrodynamics, edited by: Coronoti, S. C., and Hughes, J., vol.2, Ch.6, Gordon and Breach, New York, 55-83, 1969.

Polk, C.: Schumann resonances, CRC Handbook of Atmospherics, edited by Volland, H., vol.1, CRC Press, Bocca Raton, FL., 111178, 1982.

Price, C.: Global surface temperature and the atmospheric global circuit, Geophys. Res. Lett., 20, 1363-1366, 1993.

Satori, G. and Zieger, B.: Spectral characteristics of Schumann resonances observed in Central Europe, J. Geophys. Res., 101, $29669-29672,1996$.

Sentman, D. D. and Fraser, B. J.: Simultaneous observations of Schumann resonances in California and Australia: evidence for intensity modulation by the local height of D region, J. Geophys. Res., 96, 15 973-15 984, 1991.

Sentman, D. D.: Schumann Resonance, in Handbook of Atmospheric Electrodynamics, eited by: Volland, H., vol.1, CRC Press, Boca Raton, 267-298, 1995.

Shvets, A. V.: A technique for reconstruction of global lightning distance profile from background Schumann resonance signal, J. Atmos. Solar-terr. Phys., 63, 1061-1074, 2001.

Simpson, J. J. and Taflove, A.: Three-dimensional FDTD modeling of impulsive ELF propagation above the earth-sphere, IEEE Trans. Ant. Prop., 52 (3), 443-451, 2004.

Troyan, V. N. and Hayakawa, M.: Inverse Geophysical Problems, TERRAPUB, Tokyo, 289, 2003.

Williams, E. R.: The Schumann resonance: a global tropical thermometer, Science, 256, 1184-1187, 1992.

Williams, E. R.: Global circuit response to seasonal variations in global surface air temperature, Monthly Weather Rev., 122, 1917-1929, 1994.

Williams, E. R.: Global circuit response to temperature on distant time scales: A status report, in: Atmospheric and Ionospheric Phenomena Associated with Earthquakes, edited by: Hayakawa, M., TERRAPUB, Tokyo, 939-949, 1999.

Yang, H. and Pasko, V. P.: Three-dimensional finite-difference time-domain modeling of the Earth-ionosphere cavity resonance, Geophys. Res. Lett., 32, L03114, doi:10.1029/1004GL021343, 2005. 